\title{
Recursive objects in all finite types
}

\section{A. Grzegorczyk (Warszawa)}

1. The definition and general lemmas. The notion of recursiveness can be extended to all finite function-types in the following way. Let the natural number 0 be the type-index of the lowest type consisting of natural numbers. If $\alpha$ and $\beta$ are type-indexes, then $(\alpha \beta)=2^{\alpha}(2 \beta+1)$ is the type-index of all functions mapping the type $\alpha$ into the type $\beta$. Hence every natural number is a type-index, and the expression: the object $A$ belongs to the type $\alpha$, will be abbreviated to: $A \in \alpha$.

In the following construction we shall use the notion of application: $(\ldots, \ldots)$. If $X \in(\alpha \beta), Y \in \alpha$, then $(X, Y)$ is the value of the function $X$ for the argument $Y$ and $(X, Y) \in \beta\left(^{1}\right)$.

DEFINITION OF RECURSIVENESS (of the class $\mathcal{R}$ of the recursive objects of finite types). $\mathcal{R}$ is the smallest class satisfying two conditions:

1. If $X \in \mathcal{R}, Y \in \mathcal{R}, X \in(\alpha \beta)$ and $Y \in \alpha$, then $(X, Y) \in \mathcal{R}$.

2. The class $\mathcal{R}$ contains the following elements:

a. the number 0 of type $0(0 \in 0)$,

b. the function $S \in(00)$ (successor)

$$
(\mathcal{S}, x)=x+1
$$

c. for every $\alpha$ the element $l^{\alpha} \epsilon(\alpha \alpha)$ (identificator) such that for every $x \in \alpha\left({ }^{2}\right)$,

$$
(I, x)=x,
$$

d. for every $\alpha$ and $\beta$ the element $K^{\alpha \beta} \in(\alpha(\beta \alpha))$ (cancellator) such that for every $x \in \alpha$ and $y \in \beta$

$$
((\boldsymbol{\kappa}, x), y)=x
$$

(1) Our notation differs slightly from the notation introduced by A. Church in [2].

(2) The names "identificator", "cancellator", "compositor", "permutator", and "duplicator" are due to H. B. Curry [1]. By analogy I put "iterator" for the element $\boldsymbol{R}$. For the sake of simplicity of the formulas I omit the type indices by the symbols $I, K, B, C, D, R$. Hence in the following two occurrences of the same symbol, e.g. $D$, may denote two different duplicators. 
e. for every $\beta, \gamma, \delta$ the element $B^{\beta \gamma \delta} \epsilon((\delta \beta)((\gamma \delta)(\gamma \beta)))$ (compositor) such that for every $x \in(\delta \beta), y \in(\gamma \delta)$ and $z \in \gamma$

$$
(((B, x), y), \approx)=(x,(y, z)),
$$

f. for every $\beta, \gamma, \delta$ the element $c^{\beta \gamma \delta} \in((\beta(\gamma \delta))(\gamma(\beta \delta)))$ (permutator) such that for every $x \in(\beta(\gamma \delta)), y \in \beta, \approx \in \gamma$

$$
(((c, x), z), y)=((x, y), z),
$$

g. for every $\alpha$ and $\beta$ the element $D^{\alpha \beta} \epsilon((\alpha(\alpha \beta))(\alpha \beta))$ (duplicator) such that for every $x \in(\alpha(\alpha \beta))$ and $y \in \alpha$

$$
((D, x), y)=((x, y), y)
$$

h. for every $a$ the element $R^{a} \in(\alpha((0(\alpha \alpha))(0 \alpha)))$ (iterator) such that for every $b \in \alpha, k \in 0$ and $\theta \in(0(\alpha \alpha))$

$$
\begin{aligned}
(((R, b), c), 0) & =b, \\
(((\boldsymbol{R}, b), c),(S, k)) & =((c, k),(((R, b), c), k))\left({ }^{3}\right) .
\end{aligned}
$$

We shall deal with the class $\mathscr{P}$ of polynomials built of the function $(\ldots, \ldots)$ of application and of finite sequences $x_{1}, \ldots, x_{n}$ of objects belonging to suitable types. We shall say that these elements $x_{1}, \ldots, x_{n}$ are contained in the polynomial. $A$ is a subpolynomial of $B$ if $A, B \in \mathscr{P}$ and there exists a $C(x) \in \mathscr{P}$ such that $B=C(x / A$ ). (If we want to distinguish an object $x$ in the polynomial $W$, we write $W(x)$.) If $A, B, C$ are the subpolynomials of a polynomial $W$ and $C=(A, B)$, then for several types $\alpha$ and $\beta: A \epsilon(\alpha \beta), B \epsilon \alpha$, and $C \in \beta$. This relates of course to the elements $x_{1}, \ldots, x_{n}$ containing in the polynomial $W$ since they are subpolynomials.

(') If in the definition of the class $\mathcal{R}$ we assume the following condition $\mathrm{h}^{\prime}$. in. stead of the condition $h$. then we obtain the class of primitive reoursive objects for all types indexes at the beginning:

$\mathrm{h}^{\prime}$. for every $a$ the element $R^{+a} \in((\alpha 0)((\alpha(0(00))) \alpha(00)))$ such that for every $a \in(a 0)$ and $b \in(\alpha(0(00)))$ :

$$
\begin{aligned}
\left(\left(\left(\left(R^{+}, a\right), b\right), t\right), 0\right) & =(a, t), \\
\left(\left(\left(\left(R^{+}, a\right), b\right), t\right),(S, n)\right) & =\left(((b, t), n),\left(\left(\left(\left(R^{+}, a\right), b\right), t\right), n\right)\right) .
\end{aligned}
$$

The element $R^{+}$of course belongs to $C R$. There is an open problem whether in the definition of the class $\Re$ the element $\boldsymbol{R}^{a}$ may be replaced by the element $\boldsymbol{R}^{\prime \alpha}$ (pure
iterator) defined in 2.3 .
Let us note the following lemmas:

1.1. If $W(x)$ is a polynomial containing the element $x$, only once then there exists an $A \in \mathscr{P}$ such that $A$ does not contain $x$ and $A$ contains all elements contained in $W^{\prime}(x)$ different from $x$ and some elements from the sequences $I^{\alpha}, B^{\beta \gamma \delta}, C^{\beta \gamma \delta}$, and $A$ satisfies the equality

$$
W(x)=(A, x) \text {. }
$$

Proof by induction. (2) implies that the smallest polynomial: $x$ satisfies (8). Now suppose that (8) is satisfied by a polynomial $W(x)$. A greater polynomial $W^{i}(x)$ satisfying the supposition of our lemma may have one of two forms

$$
\begin{aligned}
& W^{\prime}(x)=(W(x), G), \\
& W^{\prime}(x)=(G, W(x)),
\end{aligned}
$$

where $G \in \mathscr{P}$ and $G$ does not contain the element $x$. In the case (i) according to (5) and (8) we have

$$
W^{\prime}(x)=((A, x), G)=(((C, A), G), x) .
$$

In the case (ii) according to (4) and (8) we have

$$
W^{\prime}(x)=(G,(A, x))=(((B, G), A), x) .
$$

Thus $W^{\prime}(x)$ also satisfies the lemma.

1.2. If $W(x)$ is a polynomial containing the element $x$ arbitrarily many times, then there exists an $A \in \mathcal{P}$ such that $A$ does not contain the element $x$, $A$ contains all elements contained in $W(x)$ and different from $x$ and some elements from the sequences $I^{\alpha}, B^{\beta \gamma \delta}, C^{\beta \gamma \delta}, D^{\alpha \beta}$, and $A$ satisfies equality (8).

Proof by induction. For the polynomials containing the element $x$ once we have Lemma 1.1. Now suppose that the lemma is true for every polynomial containing the element $x$ less than $n$ times. A polynomial $W(x)$ containing the element $x n$ times has the form: $W(x)=(F(x), G(x))$ where $F(x)$ and $G(x)$ contain the element $x$ less than $n$ times. By the induction supposition: $F(x)=(A, x)$ and $G(x)=(B, x)$. Hence

$$
\begin{aligned}
W(x) & =((A, x),(B, x))=(((C, A),(B, x)), x) \\
& =((((B,(C, A)), B), x), x)=((D,((B,(C, A)), B)), x) .
\end{aligned}
$$

THEOREM 1.3. If $W\left(x_{1}, \ldots, x_{n}\right) \in \mathscr{P}$ where $x_{1}, \ldots, x_{n}$ are all elements contained in $W\left(x_{1}, \ldots, x_{n}\right)$ arbitrarily many times and ordered in an arbitrary way, then there exists an object $F \in \mathcal{R}$ (built from the elements of the sequences $\left.I^{\alpha}, B^{\beta \gamma \delta}, C^{\beta \gamma \delta}, D^{\alpha \beta}\right)$ such that

$$
W\left(x_{1}, \ldots, x_{n}\right)=\left(\left(\left(F, x_{1}\right), x_{2}\right), \ldots, x_{n}\right) .
$$


Proof by induction with respect to the number $n$. For $n=1$ it is Lemma 1.2. Now suppose (9) for $n$. We shall prove our theorem for $n+1$. If $W^{\prime}\left(x_{1}, \ldots, x_{n}, x_{n+1}\right) \in \mathcal{P}$, then by means of Lemma 1.2 there exists a $W\left(x_{1}, \ldots, x_{n}\right) \in \mathscr{P}$ which does not contain the variable $x_{n+1}$ and is such that

(10)

$$
W^{\prime}\left(x_{1}, \ldots, x_{n}, x_{n+1}\right)=\left(W\left(x_{1}, \ldots, x_{n}\right), x_{n+1}\right) .
$$

For $W$ we have (9) by the induction hypothesis; thus from (9) and (10) we obtain (9) for $\left.W^{\prime} .{ }^{4}\right)$

1.4. If $W \epsilon \subseteq$ and all elements contained in $W$ are $R$, then the vatue of $W$ is $R$.

Proof: From Condition 1 of the definition of recursiveness.

THEOREM 1.5 (on $\lambda$-definability). If $W \in C$ and $y_{1}, \ldots, y_{k}, x_{1}, \ldots, x_{n}$ are all elements contained in $W$, and $y_{1}, \ldots, y_{k} \in \mathcal{T}$, then $\lambda x_{1}, \ldots, \lambda x_{n} W \epsilon \Re$

Proof. By Theorem 1.3 there exists an $E \in \mathcal{C}$ such that $\left.W=\left(\left(\left(E, y_{1}\right), \ldots, y_{k}\right), x\right), \ldots, x_{n}\right)$. Hence according to the meaning of the $\lambda$-operator:

$$
F=\left(\left(E, y_{1}\right), \ldots, y_{k}\right)=\lambda x_{1}, \ldots, \lambda x_{n} W .
$$

Thus $F \in \mathscr{R}$ according to 1.4 .

\section{Primitive recursive functions on natural numbers. First} let us note two lemmas on the recursive schema.

2.1 (On recursion with a parameter). If $a, b \in \mathcal{R}$, then there exists $q \in \Re$ such that

$$
\begin{gathered}
((q, t), 0)=(a, t), \\
((q, t),(\mathcal{S}, n))=(((b, t), n),((q, t), n)) .
\end{gathered}
$$

Proof. For $W(b, t, n, s)=(((b, t), n),(s, t))$ by means of 1.5 there exists a $0 \in \mathcal{R}$ such that

$$
(((b, t), n),(s, t))=\{((e, n), s), t) .
$$

Let us consider the element $p=((R, a), o) \in \mathcal{K}$. According to $(7)$ : $(p, 0)=a$ and $(p,(S, n))=((c, n),(p, n))$. Fence according to $(5)$ for $q=(C, p)$ we have

$$
\text { (13) } \quad((q, t), 0)=((p, 0), t)=(a, t)
$$

and by (12)

$$
\begin{aligned}
& \text { (14) } \begin{aligned}
(q, t),(\mathcal{S}, n)) & =\langle(p,(\mathcal{S}, n)), t)=\{((c, n),(p, n)), t) \\
& =(((b, t), n),((p, n), t))=(((b, t), n),((q, t), n)) .
\end{aligned} \\
& \text { (13) and (14) involve (11). }
\end{aligned}
$$

(4) This is a kind of completeness theorem for combinators. Cf. Curry and Feys [l]].
2.2 (on pure iteration). If $a, b \in \mathcal{L}$, then there exists $q \in \mathcal{R}$ such that $(q, 0)=a$ and $(q,(S, n))=(b,(q, n))$.

Proof. $q=((R, a),(K, b))$ according to (3) and (7) satisfies the lemma.

2.3. For ever'y a there exists an $R^{\prime \alpha} \in \mathcal{R}$ (pure iterator) such that $R^{\prime} \in(\alpha((\alpha \alpha)(0 \alpha)))$ and if $a \in \alpha$ and $b \in(\alpha \alpha)$ then

$$
\begin{gathered}
\left(\left(\left(R^{\prime}, a\right), b\right), 0\right)=a, \\
\left(\left(\left(R^{\prime}, a\right), b\right),(S, n)\right)=\left(b,\left(\left(\left(R^{\prime}, a\right), b\right), n\right)\right) .
\end{gathered}
$$

Proof. It is true for $\boldsymbol{B}^{\prime}=((\boldsymbol{C},((B, B), A)), R)$.

THEOREM 2.4. For every primitive recursive function $f$ of one variable there exists an object $F \in \Re$ such that

$$
(F, n)=f(n) .
$$

Proof. First we shall find the $\Re$-objects corresponding to some special auxillary functions: $P$ (predecessor), $+,-, \cdot, y^{x}, \sqrt{ }, E$.

$(P, 0)=0$ and $(P,(\mathcal{S}, n))=((K, n),(P, n))$ according to (3). Hence by $(7) \quad P=((\boldsymbol{R}, \mathbf{0}), \boldsymbol{K}) . \quad(+, 0)=1$ and $(+,(S, n))=((B, \mathbb{S}),(+, n))$ $=(((K,(B, S)), n),(+, n)) ;$ therefore $+=((R, I),(K,(B, S)))$, by $(7)$. Thus $((+, x), y)=x+y$. Similarly $(\dot{-}, 0)=l$ and $(\dot{-},(S, n))$ $=(((K,(B, P)), n),(\dot{-}, n))$, whence by $(7)-=((R, l),(K,(B, P)))$ and $((\dot{-}, x), y)=y \dot{-} x . \quad(\cdot, 0)=(\boldsymbol{K}, 0), \quad(\cdot,(\boldsymbol{S}, n))=(((B, D),(B,+)),(\cdot, n))$ because $((\cdot, n+1), y)=((+,((\cdot, n), y)), y)=((((B,+),(\cdot, n)), y), y)$ $=((\boldsymbol{D},((B,+),(\cdot, n))), y)$. Thus by $(7) \cdot=((\boldsymbol{R},(\boldsymbol{K}, 0)),(\boldsymbol{K},((\boldsymbol{B}, \boldsymbol{D}),(\boldsymbol{B},+))))$ and $((\cdot, x), y)=x \cdot y$. Let us put, similarly, $\operatorname{Pot}=((\boldsymbol{R},(\boldsymbol{K},(\boldsymbol{S}, 0)))$, $(K,((B, D),(B, \cdot)))) ;$ then $(($ Pot,$x), y)=y^{x}$. We put also $F_{1}=((B,($ Pot, 2$)), S)$ and $F_{2}=\left((B, S), F_{1}\right)$; then $\left(F_{2}, y\right)=(y+1)^{2}+1$. Hence: $\sqrt{0}=0$ and $\sqrt{(S, n)}=\left(\left((\sqrt{n}+1)^{2}+1\right)-(S, n)\right)+\sqrt{n}=\left(\left(+,\left((-,(S, n)),\left(F_{2}, \sqrt{n}\right)\right)\right), \sqrt{n}\right)$. According to 1.5 there exists an $F_{3}$ such that

$$
\left(\left(F_{3}, n\right), k\right)=\left(\left(+,\left((\dot{-},(S, n)),\left(F_{2}, k\right)\right)\right), k\right) .
$$

Hence $\sqrt{(S, n)}=\left(\left(F_{3}, n\right), \sqrt{n}\right)$ and $\left.\sqrt{ }^{-}=\left((R, 0), F_{3}\right)\right) . \quad E(x)=x-(\sqrt{x})_{2}$ $=\left(-,\left((\right.\right.$ Pot, 2$\left.\left.),\left(\sqrt[V]{ }^{-}, x\right)\right), x\right)$; then according to 1.5 there exists an $E$ such that $(E, x)=E(x)$. 
According to R. M. Robinson [9] the class of primitive recursive functions of natural numbers is the smallest class containing $S, E$ and closed under superposition, addition and iteration. We have our theorem for $S$ and $E$. Now suppose that $F$ satisfies (15) and similarly: $(G, n)=g(n)$; then $f(g(n))=(F,(G, n))=(((B, F), G), n) ; f(n)+g(n)=((+,(F, n)),(G, n))$ $=(H, n)$ for some $H \in \mathcal{R}$ according to 1.5 ; and $f^{n}(0)=\left(\left(\left(R^{\prime}, 0\right), F\right), n\right)$ according to 2.3 .

THEOREM 2.5. For every primitive reoursive funotion $f\left(n_{1}, \ldots, n_{k}\right)$ with $t$ arguments there exists an $F \in \mathcal{R}$ such that

$$
\left(\left(\left(F, n_{1}\right), \ldots\right), n_{k}\right)=f\left(n_{1}, \ldots, n_{k}\right) .
$$

Proof. For $f$ there exists a primitive recursive function $g$ with one argument such that

$$
f\left(n_{1}, \ldots, n_{k}\right)=g\left(\mathrm{~Pa}\left(n_{1}, \ldots, \mathrm{Pa}\left(n_{k-1}, n_{k}\right) \ldots\right)\right),
$$

where $\operatorname{Pa}(x, y)=2^{x} \cdot(2 y+1)-1$. Then

$$
\operatorname{Pa}(x, y)=((-, 1),((\cdot,((\operatorname{Pot}, x), 2)),(\$,((\cdot, 2), y)))) .
$$

Hence by 1.5 there exists a $\mathrm{Pa} \in \mathcal{R}$ such that

$$
((\mathrm{Pa}, x), y)=\mathrm{Pa}(x, y) .
$$

According to 2.4 there exists a $G \in \mathscr{R}$ such that

$$
(G, n)=g(n) \text {. }
$$

From (17)-(19) we obtain

$$
f\left(n_{1}, \ldots, n_{k}\right)=\left(G,\left(\left(\mathrm{~Pa}, n_{1}\right), \ldots,\left(\left(\mathrm{Pa}, n_{k-1}\right), n_{k}\right)\right)\right) .
$$

Thus (16) follows from 1.5.

3. Pairing functions and simultaneous recursion. Now we shall define the pairing function $\mathrm{Pa}^{\alpha \beta}$ and their converse functions Fst ${ }^{\alpha \beta}$ and $\mathrm{Snd}^{\alpha \beta}$ satisfying the equalities

$$
\begin{aligned}
& \left(\mathrm{Fst}^{\alpha \beta},\left(\left(\mathrm{Pa}^{\alpha \beta}, x\right), y\right)\right)=x \text { for } \quad x \in \alpha, y \in \beta \text {, } \\
& \left(\mathrm{Snd}^{\alpha \beta},\left(\left(\mathrm{Pa}^{\alpha \beta}, x\right), y\right)\right)=y \quad \text { for } \quad x \in \alpha, y \in \beta .
\end{aligned}
$$

First we define a natural function $\varphi(n, k)$ such that

$$
\left(\left(\mathrm{Pa}^{\alpha \beta}, x\right), y\right) \in \varphi(\alpha, \beta) \quad \text { for } \quad x \in \alpha, y \in \beta \text {. }
$$

Hence the types of these elements will be the following:

$$
\begin{gathered}
\mathrm{Pa}^{\alpha \beta} \epsilon(\alpha(\beta(\varphi(\alpha, \beta)))), \\
\operatorname{Fst}^{\alpha \beta} \epsilon(\varphi(\alpha, \beta) \alpha), \\
\operatorname{Snd}^{\alpha \beta} \in(\varphi(\alpha, \beta) \beta) .
\end{gathered}
$$

We put

$$
\begin{aligned}
& \varphi(0, \alpha)=\alpha, \\
& \varphi(\alpha, 0)=\alpha,
\end{aligned}
$$$$
\varphi((\alpha \beta),(\gamma \delta))=(\varphi(\alpha, \gamma) \varphi(\beta, \delta)) .
$$

First we need the constant function for every type:

$$
0_{0}=0 \text {, }
$$

$0_{(\alpha \beta)}=\lambda x 0_{\beta} \quad$ for every $x \in \alpha$,

and the pairing functions for natural numbers:

$$
\begin{gathered}
\mathrm{Pa}^{00}=\mathrm{Pa} \quad(\text { defined in }(18)), \\
\mathrm{Fst}^{00}=\lambda z \exp (2, z+1),\left(^{5}\right) \\
\mathrm{Snd}^{00}=\lambda z\left[\frac{\left[\frac{z+1}{2^{(\mathrm{Fst}, z)}}\right]-1}{2}\right] .
\end{gathered}
$$

They belong to $R$ according to 2.5 and satisfy (20) and (21). Now we define others inductively:

Every type a can be represented in the form:

$$
\begin{aligned}
& a=\left(a_{1}\left(\ldots\left(a_{n} 0\right) \ldots\right)\right), \\
& \mathrm{Pa}^{0 a}=\lambda x_{0} \lambda f_{a} \lambda y_{a_{1}} \ldots \lambda y_{a_{n}}\left(\left(\mathrm{~Pa}^{00}, x\right),\left(\left(f_{\alpha}, y_{a_{1}}\right), \ldots, y_{a_{n}}\right)\right), \\
& \mathrm{Pa}^{\alpha 0}=\lambda f_{a} \lambda x_{0} \lambda y_{\alpha_{1}} \ldots \lambda y_{a_{n}}\left(\left(\mathrm{~Pa}^{00},\left(\left(f_{\alpha}, y_{a_{1}}\right), \ldots, y_{a_{n}}\right)\right), x_{0}\right), \\
& \mathrm{Fst}^{0 a}=\lambda g_{\alpha}\left(\mathrm{Fst}^{(00)},\left(\left(g_{a}, 0_{\alpha_{1}}\right), \ldots, 0_{a_{n}}\right)\right), \\
& \operatorname{Snd}^{0 a}=\lambda g_{a} \lambda y_{a_{1}} \ldots \lambda y_{a_{n}}\left(\operatorname{Snd}^{00},\left(\left(g_{a}, y_{a_{1}}\right), \ldots, y_{a_{n}}\right)\right) \text {, } \\
& \mathrm{Fst}^{a 0}=\lambda g_{a} \lambda y_{\alpha_{1}} \ldots \lambda y_{a_{n}}\left(\mathrm{Fst}^{00},\left(\left(g_{a}, y_{a_{1}}\right), \ldots, y_{a_{n}}\right)\right) \text {, } \\
& \operatorname{Snd}^{\alpha 0}=\lambda g_{a}\left(\operatorname{Snd}^{00},\left(\left(g_{a}, 0_{a_{1}}\right), \ldots, 0_{a_{n}}\right)\right), \\
& \mathrm{Pa}^{(\alpha \beta)(\gamma \delta)}=\lambda x_{(\alpha \beta)} \lambda y_{(\gamma \delta)} \lambda z_{\varphi(\alpha \gamma)}\left(\left(\mathrm{Pa}^{\beta \delta},\left(x,\left(\operatorname{Fst}^{\alpha \gamma}, z\right)\right)\right),\left(y,\left(\operatorname{Snd}^{\alpha \gamma}, z\right)\right)\right), \\
& \mathrm{Fst}^{(\alpha \beta)(\gamma \delta)}=\lambda g_{\varphi((a \beta),(\gamma \delta))} \lambda u_{\alpha}\left(\mathrm{Fst}^{\beta d},\left(g,\left(\left(\mathrm{~Pa}^{\alpha \gamma}, u\right), 0_{\gamma}\right)\right)\right), \\
& \operatorname{Snd}^{(\alpha \beta)(\gamma \delta)}=\lambda g_{\varphi((a \beta),(\gamma \delta))} \lambda u_{\gamma}\left(\operatorname{Snd}^{\beta \delta},\left(g,\left(\left(\mathrm{~Pa}^{\alpha \gamma}, 0_{\alpha}\right), u\right)\right)\right) .
\end{aligned}
$$

(5) $\exp (2, x)=$ the exponent $e$ such that $2^{e} \mid x$ and $\sim\left(2^{e+1} \mid x\right)$. 
THEOREM 3.1. The elements $\mathrm{Pa}^{\alpha \beta}, \mathrm{Fst}^{\alpha \beta}$ and $\mathrm{Snd}^{\alpha \beta}$ belong to $\mathcal{R}$ and satisfy (20) and (21).

Proof. It is evident that these elements satisfy (21) and by 1.5 they belong to $\mathcal{R}$. It remains to prove (20). The proof is inductive. For the pair of types 00 it is the familiar property of pairing functions. Now we examine the pair $0 \alpha$ and $\alpha 0$ :

$$
\begin{aligned}
\left(\mathrm{Fst}^{0 \alpha},\left(\left(\mathrm{Pa}^{0 \alpha}, x_{0}\right), f_{a}\right)\right) & =\left(\mathrm{Fst}^{00},\left(\left(\mathrm{~Pa}^{00}, x_{0}\right),\left(\left(f_{a}, 0_{a_{1}}\right), \ldots, 0_{a_{n}}\right)\right)\right)=x_{0_{0}}, \\
\left(\operatorname{Snd}^{0 \alpha},\left(\left(\mathrm{Pa}^{0 \alpha}, x_{0}\right), f_{a}\right)\right) & =\lambda y_{a_{1}} \ldots \lambda y_{a_{n}}\left(\operatorname{Snd}^{00},\left(\left(\mathrm{~Pa}^{00}, x_{0}\right),\left(\left(f_{a}, y_{a_{1}}\right), \ldots, y_{a_{n}}\right)\right)\right) \\
& =\lambda y_{a_{1}} \ldots \lambda y_{a_{n}}\left(\left(f_{\alpha}, y_{a_{1}}\right), \ldots, y_{a_{n}}\right)=f_{a}, \\
\left(\mathrm{Fst}^{\alpha 0},\left(\left(\mathrm{~Pa}^{a 0}, f_{a}\right), x_{0}\right)\right) & =\lambda y_{a_{1}} \ldots \lambda y_{a_{n}}\left(\mathrm{Fst}^{00},\left(\left(\mathrm{~Pa}^{00},\left(\left(f_{a}, y_{a_{1}}\right), \ldots, y_{a_{n}}\right)\right), x_{0}\right)\right) \\
& =\lambda y_{a_{1}} \ldots \lambda y_{a_{n}}\left(\left(f_{a}, y_{a_{1}}\right), \ldots, y_{a_{n}}\right)=f_{a}, \\
\left(\operatorname{Snd}^{\alpha 0},\left(\left(\mathrm{~Pa}^{a 0}, f_{a}\right), x_{0}\right)\right) & \left.\left.=\left(\operatorname{Snd}^{00},\left(\left(\mathrm{~Pa}^{00},\right)\left(f, 0_{a_{1}}\right), \ldots, 0_{a_{n}}\right)\right), x_{0}\right)\right)=x_{0} .
\end{aligned}
$$

Then suppose that $(20)$ is true for the pairs $\alpha \gamma$ and $\beta \delta$; we shall prove that $(20)$ is true for the pair $(\alpha \beta)(\gamma \delta)$ :

$$
\begin{aligned}
& \left(\mathrm{Fst}^{(\alpha \beta)(\gamma \delta)},\left(\left(\mathrm{Pa}^{(\alpha \beta)(\gamma \delta)}, x_{(\alpha \beta)}\right), y_{(\gamma \delta)}\right)\right) \\
& =\lambda u_{a}\left(\mathrm{Fst}^{\beta \delta},\left(\lambda z_{q(\alpha \gamma)}\left(\left(\mathrm{Pa}^{\beta \delta},\left(x,\left(\operatorname{Fst}^{\alpha \gamma}, z\right)\right)\right),\left(y,\left(\operatorname{Snd}^{\alpha \gamma}, z\right)\right)\right),\left(\left(\operatorname{Pa}^{\alpha{ }^{\alpha}}, u_{a}\right), 0_{\gamma}\right)\right)\right) \\
& =\lambda u_{a}\left(\mathrm{Fst}^{\beta \delta},\left(\left(\mathrm{Pa}^{\beta \delta},\left(x,\left(\mathrm{Fst}^{\alpha \gamma},\left(\left(\mathrm{Pa}^{\alpha \gamma}, u_{a}\right), 0_{\gamma}\right)\right)\right)\right),\left(y,\left(\operatorname{Snd}^{\alpha \gamma},\left(\left(\mathrm{Pa}^{\alpha \nu}, u_{a}\right), 0_{\gamma}\right)\right)\right)\right)\right) \\
& =\lambda u_{a}\left(\mathrm{Fst}^{\beta \delta},\left(\left(\mathrm{Pa}^{\beta \delta},\left(x, u_{a}\right)\right),\left(y, 0_{\gamma}\right)\right)\right)=\lambda u_{\alpha}\left(x, u_{\alpha}\right)=x, \\
& \left(\operatorname{Snd}^{(\alpha \beta)(\gamma \delta)},\left(\left(\mathrm{Pa}^{(\alpha \beta \beta)(\gamma \delta)}, x_{(\alpha \beta)}\right), y_{(\gamma \delta))}\right)\right. \\
& =\lambda u_{\gamma}\left(\operatorname{Snd}^{\beta \delta},\left(\lambda z_{\varphi(\alpha \gamma)}\left(\left(\operatorname{Pa}^{\beta \delta},\left(x,\left(\operatorname{Fst}^{\alpha \gamma}, z\right)\right)\right),\left(y,\left(\operatorname{Snd}^{\alpha \gamma \gamma}, z\right)\right)\right),\left(\left(\mathbf{P a}^{\alpha \gamma}, \mathbf{O}_{a}\right), u_{\nu}\right)\right)\right) \\
& =\lambda u_{\gamma}\left(\operatorname{Snd}^{\beta \delta},\left(\left(\mathrm{Pa}^{\beta \delta},\left(x,\left(\mathrm{Fst}^{\alpha \gamma},\left(\left(\mathrm{Pa}^{\alpha \gamma}, 0_{a}\right), u_{\gamma}\right)\right)\right)\right),\left(y,\left(\operatorname{Snd}^{\alpha \gamma},\left(\left(\mathrm{Pa}^{\alpha \gamma}, 0_{\alpha}\right), u_{\gamma}\right)\right)\right)\right)\right) \\
& \left.=\lambda u_{\gamma}\left(\operatorname{Snd}^{\beta \delta},\left(\mathbf{P a}^{\beta \delta},\left(x, 0_{\alpha}\right)\right),\left(y, u_{\gamma}\right)\right)\right)=\lambda u_{\gamma}\left(y, u_{\gamma}\right)=y \text {. }
\end{aligned}
$$

Thus $(20)$ is true for all pairs $\alpha \beta$.
THEOREM 3.2 (on simultaneous recursion). If $a_{1}, \ldots, a_{n} \in \mathcal{R}$ and $a_{i} \in a_{i}, p_{1}, \ldots, p_{n} \in \mathcal{R}$ and $p_{i} \in\left(\alpha_{1}\left(\ldots\left(\alpha_{n} \alpha_{i}\right) \ldots\right)\right)$, then $q_{1}, \ldots, q_{n} \in \mathcal{R}$ and $q_{i} \epsilon\left(0 \alpha_{i}\right)$ where $q_{i}$ are defined as follows:

$$
\begin{aligned}
\left(q_{i}, 0\right) & =a_{i}, \\
\left(q_{i},(S, k)\right) & =\left(\left(p_{i},\left(q_{1}, k_{i}\right)\right), \ldots,\left(q_{n}, k\right)\right) .
\end{aligned}
$$

Proof. We define by means of pure iteration a function $g$ :

$$
\begin{aligned}
(g, 0) & =\left(\left(\mathrm{Pa}, a_{1}\right), \ldots\left(\left(\mathrm{Pa}, a_{n-1}\right), a_{n}\right)\right), \\
(g,(S, k)) & =\left(\left(\mathrm{Pa}, r_{1}\right), \ldots\left(\left(\mathrm{Pa}, r_{n-1}\right), r_{n}\right)\right)
\end{aligned}
$$

where

$r_{i}=\left(\ldots\left(\left(p_{i},(\right.\right.\right.$ Fst,$\left.(g, k))\right),($ Fst,$($ Snd,$\left.(g, k)))\right), \ldots($ Snd,$\ldots($ Snd,$\left.(g, k)) \ldots)\right)$. By 2.2 and $3.1 g \in \Re$ and

$$
\begin{aligned}
\left(q_{1}, k\right) & =(\text { Fst },(g, k)) \\
\left(q_{2}, k\right) & =(\text { Fst },(\text { Snd },(g, k))) \\
\ldots \ldots & \ldots \ldots \\
\left(q_{n-1}, k\right) & =(\text { Fst },(\text { Snd }, \ldots(\text { Snd },(g, k)) \ldots)), \\
\left(q_{n}, k\right) & =(\operatorname{Snd},(\operatorname{Snd}, \ldots(\operatorname{Snd},(g, k)) \ldots)) .
\end{aligned}
$$

4. An equivalent definition. The class $R$ may be characterized as having only $0, S$ and $I^{\alpha}$ as generators and being closed under six operations.

THeonsm 4.1. $\mathcal{R}$ is the smallest class containing $0, \mathbf{S}$, and $I^{a}$ for every type a and closed under the following operations:

a. if $I F \in \mathcal{R} \cap \alpha$, then there exists a $G \in \Re$ such that for every $x \in \beta$

$$
(G, x)=H^{\prime}
$$

b. if $F \in R \cap(\alpha(\beta(\gamma \delta)))$ then there exists a $G \in R \cap(\alpha(\gamma(\beta \delta)))$ such that for any $x \in \alpha, y \in \beta, \approx \epsilon \gamma$

$$
(((G, x), z), y)=(((x, x), y), z) ;
$$

c. for every $H^{\prime} \in \mathcal{R} \cap(\gamma \delta)$ and $G \in \mathcal{R} \cap(\alpha(\beta \gamma))$ there exists an $H \in \mathcal{R} \cap$ $\cap(\alpha(\beta \delta))$ such that for every $x \in \alpha$ and $y \in \beta$

$$
((H, x), y)=(F,((G, x), y)) ;
$$

Fundamenta Mathematicae, T. LIV 
d. for every $F \in \mathcal{R} \cap(\alpha(\beta(\beta \gamma)))$ there exists a $D \in \mathcal{R} \cap(\alpha(\beta \gamma))$ such that for every $x \in \alpha$ and $y \in \beta$

$$
((D, x), y)=(((F, x), y), y) ;
$$

e. for every $F \in \mathcal{R} \cap(\alpha \beta)$ and $G \in \mathcal{R} \cap(\alpha(0(\beta \beta)))$ there exists an $H \in R \cap(\alpha(0 \beta))$ such that for any $t \in a$ and $n \in N$

$$
((H, t), 0)=\left(H^{\prime}, t\right)
$$

$$
((H, t),(S, n))=(((G, t), n),((H, t), n)) ;\left(^{(0)}\right)
$$

f. for every $F \in \Re \cap(\alpha \beta)$ and $G \in \mathcal{R} \cap \alpha,(F, G) \in \mathcal{R}$.

Proof. From 1.5 and 2.1 it follows immediately that the class $\propto$ satisfies the conditions a.-f. Conversely starting from a.-f. we can construct the elements $K^{\alpha \beta}, B^{\alpha \beta \gamma}, C^{\alpha \beta \gamma}, D^{\alpha \beta}$, and $R^{\alpha}$.

$C$. From $b$. it follows that for $/$ there exists a $C \in C R$ such that by (21)

$$
(((c, x), z), y)=(((I, x), y), z)=((x, y), z) .
$$

$B$. From the preceding result, c., b. and $f$, it follows that

$$
\begin{aligned}
(x,(y, z)) & =((I, x),((I, y), z))=(((C, I),((I, y), z)), x) \\
& =(((H, y), z), x)=\mid((G, y), x), z)=\left(\left(\left(\left(C, G^{\prime}\right), x\right), y\right), z\right) \\
& =(((B, x), y), z), \quad \text { for } \quad B=(C, G) .
\end{aligned}
$$

$\kappa$. From a. we infer that for $I$ there exists a $G$ such that $(G, y)=I$. Hence

$x=(I, x)=((G, y), x)=(((C, G), x), y)=((\kappa, x), y) \quad$ for $\quad k=x(c, G)$.

$D$. From d. it follows that for $l$ there exists a $D$ such that

$$
((0, x), y)=(((I, x), y), y)=((x, y), y) \text {. }
$$

R. For every $\alpha$ the element $R^{\alpha}$ may be constructed in the following way. First let us note that using schema (29) we can obtain all primitive recursive functions on natural numbers. Thits also $\mathrm{Pa}^{(00)}$, Fst ${ }^{(00)}$, and $\mathrm{Snd}^{(00)}$. Hence by means of the preceding results involving 1.5 we find that for every type $\alpha$ and $\beta$ the elements $\mathrm{Pa}^{(\alpha \beta)}, \mathrm{Fst}^{(\alpha \beta)}, \mathrm{Snd}^{\alpha \beta}$ defined in (23) belong to $R$. According to 3.1 for the types $\alpha$ and $\beta=(0(\alpha \alpha))$

(9) If we assume the condition e. only for $\beta=0$, then wo obtain the class of primitive recursive objects mentioned in footnote $\left({ }^{(9)}\right.$. the elements $\mathrm{Pa}^{(\alpha \beta)}, \mathrm{Fst}^{(\alpha \beta)}$, Snd ${ }^{(\alpha \beta)}$ satisfy $(20)$, and (22). e. implies that for $F=$ Fst $^{(\alpha a)}$ and $G=$ Snd $^{(\alpha \beta)}$ there exists an $H \in \mathcal{R}$ satisfying (29). From (22) and (29) it follows that for every $b \in \alpha$ and $c \in(0(\alpha \alpha))$ :

$$
\begin{aligned}
((H,((\mathrm{~Pa}, b), c)), 0) & =b, \\
((H,((\mathrm{~Pa}, b), c)),(\boldsymbol{S}, n)) & =((c, n),((H,((\mathrm{~Pa}, b), c)), n)) .
\end{aligned}
$$

Hence using $B$ we prove inductively that the element $R=((B,(B, H)), \mathrm{Pa})$ satisfies (7), because

$$
\begin{aligned}
((\boldsymbol{R}, b), c) & =((((B,(B, H)), \mathrm{Pa}), b), c)=\mid((B, H),(\mathrm{Pa}, b)), c) \\
& =(H,((\mathrm{~Pa}, b), c)) \cdot\left(^{(7}\right)
\end{aligned}
$$

5. Recursive functionals taking natural values. Now we shall consider a class of recursive functionals $F\left(x_{1}, \ldots, x_{n}\right)=k$ of many arguments assuming only natural numbers as values. The types considered will be the following: $0=$ the type of natural numbers; if $\tau_{1}, \ldots, \tau_{n}$ are types, then $\left\langle\tau_{1}, \ldots, \tau_{n}\right\rangle$ is the type of all functions of $n$ arguments mapping the product $\tau_{1} \times \ldots \times \tau_{n}$ in natural numbers. We shall define the recursive functionals for all these types.

5.1 (The definition). The class Rec is the smallest class which contains $0,0(x)=0, S(x)=x+1$, and for every type $\tau=\left\langle\tau_{1}, \ldots, \tau_{n}\right\rangle$, the element $I^{x}$ for which

$$
I\left(X_{\tau}, X_{\tau_{1}}, \ldots, X_{\tau_{n}}\right)=X_{\tau}\left(X_{\tau_{1}}, \ldots, X_{\tau_{n}}\right),
$$

and is closed under the following conditions:

a. if $F \in \operatorname{Rec} \cap \tau$ and $\tau=\left\langle\tau_{1}, \ldots, \tau_{n}\right\rangle$, then there exists a $G \in \operatorname{Rec}$ such that for every $x \in \beta$ and $y_{1}, \ldots, y_{n}\left(y_{i} \in \tau_{i}\right)$ :

$$
G\left(x, y_{1}, \ldots, y_{n}\right)=F\left(y_{1}, \ldots, y_{n}\right),
$$

b. if $F \in \operatorname{Rec} \cap \tau$ and $\tau=\left\langle\pi, \varrho, \sigma, \tau_{1}, \ldots, \tau_{n}\right\rangle$ then there exists a $G \in \operatorname{Rec}$ such that $G \in\left\langle\pi, \sigma, \varrho, \tau_{1}, \ldots, \tau_{n}\right\rangle$ and for all elements $x, y, z$, $u_{1}, \ldots, u_{n}$ of suitable types:

$$
G\left(x, z, y, u_{1}, \ldots, u_{n}\right)=F^{\prime}\left(x, y, z, u_{1}, \ldots, u_{n}\right) ;
$$

this condition comprises the case in which $F$ has only three arguments $\tau=\langle\pi, \varrho, \sigma\rangle$ formula (32) then assumes the form:

$$
G(x, z, y)=F(x, y, z) \text {; }
$$

(7) Proving the analogon of 4.1 for the class of primitive recursive objects mentioned in footnotes $\left(^{8}\right)$ and $\left(^{(}\right)$we construct the element $R^{+}$joining the parameters $a, b, t$ of $\mathrm{h}^{\prime}$. in one by using the pairing functions. 
c. for every $F \in \operatorname{Rec} \cap \tau$ and $G \in \operatorname{Rec} \cap\left\langle\pi, \varrho, \sigma_{1}, \ldots, \sigma_{n}\right\rangle$, if $\tau=\left\langle\sigma, \tau_{1}, \ldots, \tau_{k}\right\rangle$ and $\sigma=\left\langle\sigma_{1}, \ldots, \sigma_{n}\right\rangle$, then there exists an $H \in \operatorname{Rec} \cap\left\langle\pi, \varrho, \tau_{1}, \ldots, \tau_{k}\right\rangle$ such that for all elements of suitable types:

(33) $\quad H\left(x, y, u_{1}, \ldots, u_{k}\right)=\mathbb{H}\left(\lambda z_{1} \ldots \lambda z_{n} G\left(x, y, z_{1}, \ldots, z_{n}\right), u_{1}, \ldots, u_{k}\right)$;

this condition comprises also the cases in which $\tau=\langle\sigma\rangle$ or $G \epsilon\langle\pi, \varrho\rangle$ and $\sigma=0$; this gives three special cases:

$\left(33^{\prime}\right) \quad H(x, y)=F\left(\lambda z_{1} \ldots \lambda z_{n} G\left(x, y, z_{1}, \ldots, z_{n}\right)\right)$

$\left(33^{\prime \prime}\right) \quad H\left(x, y, u_{1}, \ldots, u_{k}\right)=F\left(G(x, y), u_{1}, \ldots, u_{k}\right)$,

$\left(33^{\prime \prime \prime}\right) \quad H(x, y)=F(G(x, y))$;

d. for every $F \in \operatorname{Rec} \cap\left\langle\pi, \varrho, \varrho, \tau_{1}, \ldots, \tau_{n}\right\rangle$ there existis an $H \in$ Rec $n$ $\cap\left\langle\pi, \varrho, \tau_{1}, \ldots, \tau_{n}\right\rangle$ such that

$$
H\left(x, y, u_{1}, \ldots, u_{n}\right)=F\left(x, y, y, u_{1}, \ldots, u_{n}\right) \text {, }
$$

this condition comprises also the case in which $F$ has only three arguments $F \in\langle\pi, \varrho, \varrho\rangle$ and

$$
H(x, y)=H(x, y, y)
$$

e. for every $F \in \operatorname{Rec} \cap\left\langle\pi, \tau_{1}, \ldots, \tau_{n}\right\rangle$ and $G \in \operatorname{Rec} \cap\left\langle\pi, 0,\left\langle\tau_{1}, \ldots, \tau_{n}\right\rangle\right\rangle$ there exists an $H \in \operatorname{Rec} \cap\left\langle\pi, 0, \tau_{1}, \ldots, \tau_{n}\right\rangle$ such that

$$
\begin{aligned}
H\left(t, 0, u_{1}, \ldots, u_{n}\right) & =F\left(t, u_{1}, \ldots, u_{n}\right), \\
H\left(t, n+1, u_{1}, \ldots, u_{n}\right) & =G\left(t, n, \lambda u_{1} \ldots \lambda u_{n} H\left(t, n, u_{1}, \ldots, u_{n}\right), u_{1}, \ldots, u_{n}\right) ;
\end{aligned}
$$

this condition comprises also the case in which $F$ has only one argument $F \in\langle\pi\rangle$; then schema (35) has the form:

$$
\begin{aligned}
H(t, 0) & =F(t), \\
H(t, n+1) & =G(t, n, H(t, n)) ;\left({ }^{8}\right)
\end{aligned}
$$

f. for every $F \in \operatorname{Rec} \cap\left\langle\pi, \tau_{1}, \ldots, \tau_{n}\right\rangle$ and $G \in \operatorname{Rec} \cap \pi$ there exists $H \in \operatorname{Rec} \cap\left\langle\tau_{1}, \ldots, \tau_{n}\right\rangle$ such that

$$
H\left(u_{1}, \ldots, u_{n}\right)=F\left(G, u_{1}, \ldots, u_{n}\right) ;
$$

this conditions comprises also the trivial case in which $I^{r}$ is a function of one argument $F^{\prime} \in\langle\pi\rangle$ and $H$ is a natural number:

$$
H=F(G) \text {. }
$$

(8) In order to obtain the definition of the class of primitive recursive functionals for all types described at the beginning of Section 5 we assume only the special case $\left(35^{\prime}\right)$ of the induction schema instead of (35).
Remark. We do not need to specify certain other special cases, which are natural with regard to the expected analogy with the characterization of the class $\mathcal{R}$ in 4.1. (This analogy will be the object of theorem 5.2.) All natural special cases follow from those mentioned in the definition. First the function $I^{0}(x)=x$ may be defined by using $\left(35^{\prime}\right)$, (36) and (31): namely $I^{0}(n)=I(0, n)$ where

$$
\begin{aligned}
I(t, 0) & =0(t), \\
I(t, n+1) & =S(t, n, I(t, n)),
\end{aligned}
$$

where $S(t, n, y)=S(n, y)=S(y)$ according to $(31)$.

As regards condition a. if $\tau=0$ schema (31) gives the constant functions $G_{k}(x)=k$. But every $G_{k}$ may be defined by using $0(x),(31)$, (36) and $\left(33^{\prime \prime \prime}\right)$. Of course $G_{k}(x)=G_{k}(0, x)$, where $G_{k}(t, x)=\underbrace{S(\ldots S}_{k}(0(t, x)))$.

As regards condition $b$. it may be remarked that in order to permute the later arguments (not only the second with the thrid, as is allowed by (32)) we must first define the pairing functions and using them join the preceding arguments in one.

It may be remarked also that the function $I^{0}(n)=n$, as well as the other primitive recursive functions of natural numbers, may be obtained by using schema (35) without using the simplified form $\left(35^{\prime \prime}\right)$. Hence without $\left(35^{\prime}\right)$ we can obtain the pairing functions and we can show that $\left(35^{\prime}\right)$ may be reduced to $(35)$ according to the following idea: if $H$ is defined by $\left(35^{\prime}\right)$, then $H(t, n)=H(t, n, 0)$ where: $H(t, 0, u)=F(t)$, and $H(t, n+1, u)=G\left(t, n, I^{\langle 0\rangle}(\lambda u H(t, n, u), u)\right)$.

Now we shall compare the present notion with the previous one. We shall show that they are isomorphic. First it is easy to see that there exists a one-to-one correspondence $\Psi$ between all functions of many arguments assuming natural values which we are considering and all functions of one argument considered in the preceding sections. First we establish a one-to-one correspondence between the types:

$$
\tau(0)=0,
$$

if $\alpha=\left(a_{1}\left(\ldots\left(a_{n} 0\right) \ldots\right)\right)$ then $\tau(\alpha)=\left\langle\tau\left(a_{1}\right), \ldots, \tau\left(a_{n}\right)\right\rangle\left(\alpha, a_{1}, \ldots, \alpha_{n}\right.$ will remain for the types described in Section 1$)$.

For $n \in 0$ we put $\Psi(n)=n$. Now, if $f$ is a function of one argument, $f \in \alpha$ according to the notation of Section 1 and $\alpha=\left(\alpha_{1}\left(\ldots\left(\alpha_{n} 0\right) \ldots\right)\right)$, then the corresponding element $F=\Psi(f)$ is defined as follows:

$$
\Psi(f)\left(X_{\tau\left(a_{1}\right)}, \ldots, X_{\tau\left(a_{n}\right)}\right)=\left(\left(f, \Psi^{-1}\left(X_{\tau\left(a_{1}\right)}\right)\right), \ldots, \Psi^{-1}\left(X_{\tau\left(a_{n}\right)}\right)\right) .
$$

THEOREM 5.2. $f \in R \equiv \Psi(f) \in$ Rec. 
Proof. We use the definition of $\mathcal{R}$ given by 4.1. The mapping $\Psi$ transforms the initial elements $0, S, I^{a}$ of the class $\mathcal{R}$ into the initial functionals $0, S, I^{\tau(\alpha)}$ of the class Rec. It is evident that $0=\Psi(0)$ and $S=\Psi(S)$. The formula $\Psi\left(I^{\alpha}\right)=I^{\tau(\alpha)}$ follows from (37), (2) and $(30)$ : $\Psi\left(I^{a}\right)\left(X_{\tau(\alpha)}, X_{\tau\left(\alpha_{1}\right)}, \ldots, X_{\tau\left(a_{n}\right)}\right)=\left(\left(\left(I^{a}, \Psi^{-1}\left(X_{\tau(\alpha)}\right)\right), \Psi^{-1}\left(X_{\tau\left(a_{1}\right)}\right)\right), \ldots, \Psi^{-1}\left(X_{\tau\left(a_{n}\right)}\right)\right)$ $=\left(\left(\Psi^{-1}\left(X_{\tau(\alpha)}\right), \Psi^{-1}\left(X_{\tau\left(\alpha_{1}\right)}\right)\right), \ldots, \Psi^{-1}\left(X_{\tau\left(\alpha_{n}\right)}\right)\right)=\Psi\left(\Psi^{-1}\left(X_{\tau(\alpha)}\right)\right)\left(X_{\tau\left(\alpha_{1}\right)}, \ldots, X_{\tau\left(a_{n}\right)}\right)$ $=X_{\tau(\alpha)}\left(X_{\tau\left(a_{1}\right)}, \ldots, X_{\tau\left(a_{n}\right)}\right)=I^{\tau(\alpha)}\left(X_{\tau(\alpha)}, X_{\tau\left(\alpha_{1}\right)}, \ldots, X_{\tau\left(a_{n}\right)}\right)$.

Now we shall prove that an element $H \in \mathcal{C}$ is obtained from $F^{1}$ and $G$ by means of one of the operations a.-f. mentioned in 4.1 if and only if $\Psi(B)$ is obtained from $\Psi(F)$ and $\Psi(G)$ by means of the corresponding operation a.-f. mentioned in 5.1

a. If $F \in \mathcal{R} \cap \alpha$ and $\Psi\left(F^{\prime}\right) \in$ Rec besides $G$ satisfies (25) and $G^{\prime}$ satisfies (31) with the substitution $F / \Psi\left(F^{\prime}\right)$, then $G^{\prime}=\Psi(G)$. Indeed, if $a=\left(\alpha_{1}\left(\ldots\left(\alpha_{n} 0\right)\right)\right)$, then (25), (37) and (31) imply that $\left(\left((G, x), y_{1}\right), \ldots, y_{n}\right)$ $=\left(\left(F, y_{1}\right), \ldots, y_{n}\right)=\Psi(F)\left(\Psi\left(y_{1}\right), \ldots, \Psi\left(y_{n}\right)\right)=G^{\prime}\left(\Psi(x), \Psi\left(y_{1}\right), \ldots, \Psi\left(y_{n}\right)\right)$, whence by (37) $G^{\prime}=\Psi(G)$.

b. If $F \in \Re \cap(\alpha(\beta(\gamma \delta))), \delta=\left(\delta_{1}\left(\ldots\left(\delta_{n} 0\right)\right)\right), \Psi(F) \in$ Rec, $G$ satisfies $(26)$ and $G^{\prime}$ satisfies (32) with the substitution $F / \Psi(F)$, then $G^{\prime}=\Psi(G)$. Indeed, (26), (37) and (32) imply that

$$
\begin{aligned}
\left.\left((((G, x), z), y), u_{1}\right), \ldots, u_{n}\right) & =\left(\left((((F, x), y), z), u_{1}\right), \ldots, u_{n}\right) \\
& =\Psi(F)\left(\Psi(x), \Psi(y), \Psi(z), \Psi\left(u_{1}\right), \ldots, \Psi\left(u_{n}\right)\right) \\
& =G^{\prime}\left(\Psi(x), \Psi(z), \Psi(y), \Psi\left(u_{1}\right), \ldots, \Psi\left(u_{n}\right)\right),
\end{aligned}
$$

whence according to (37) $G^{\prime}=\Psi(G)$.

c. If $F \in \mathcal{R} \cap(\gamma \delta), G \in P \cap(\alpha(\beta \gamma)), H$ satisfies $(27), \delta=\left(\delta_{1}\left(\ldots\left(\delta_{k} 0\right)\right)\right)$, $\Psi(F) \in \operatorname{Rec}, \Psi(G) \in \operatorname{Rec}$, and $H^{\prime}$ satisfies (33) with the substitution $W^{\prime} / \Psi\left(I^{\prime}\right)$ and $G / \Psi(G)$, then $H^{\prime}=\Psi(H)$. First let us note that (37) implies the equalities

$$
\begin{aligned}
\Psi\left(\lambda z_{1} \ldots \lambda z_{n}\right. & \left.\left.\left(((G, x), y), z_{1}\right), \ldots z_{n}\right)\right)\left(Z_{1}, \ldots, Z_{n}\right) \\
& \left.=\left(\left(\lambda z_{1} \ldots \lambda z_{n}\left(((G, x), y), z_{1}\right), \ldots z_{n}\right), \Psi^{-1}(Z)\right), \ldots, \Psi\left(Z_{n}\right)\right) \\
& =\left(\left(((G, x), y), \Psi^{-1}\left(Z_{1}\right)\right), \ldots, \Psi^{-1}\left(Z_{n}\right)\right) \\
& =\Psi(G)\left(\Psi(x), \Psi(y), Z_{1}, \ldots, Z_{n}\right) \\
& =\lambda Z_{1} \ldots \lambda Z_{n} \Psi(G)\left(\Psi(x), \Psi(y), Z_{1}, \ldots, Z_{n}\right)\left(Z_{1}, \ldots, Z_{n}\right)
\end{aligned}
$$

hence

(38) $\Psi\left(\lambda z_{1} \ldots \lambda z_{n}\left(((G, x), y), z_{1}\right), \ldots z_{n}\right)$

$$
\left.=\lambda Z_{1} \ldots \lambda Z_{n} \Psi(G) \Psi(x), \Psi(y), Z_{1}, \ldots, Z_{n}\right) .
$$

Now if $\gamma=\left(\gamma_{1}\left(\ldots\left(\gamma_{n} 0\right)\right)\right)$ the formulas $(27),(37),(38)$ and (33) imply the equalities

$$
\begin{aligned}
& \left.\left(((H, x), y), u_{1}\right), \ldots, u_{k}\right)=\left(\left((F,((G, x), y)), u_{1}\right), \ldots, u_{k}\right) \\
& =\left(\left(\left(F, \lambda z_{1} \ldots \lambda z_{n}\left(\left(((G, x), y), z_{1}\right), \ldots, z_{n}\right)\right), u_{1}\right), \ldots, u_{k}\right) \\
& \left.=\Psi\left(F^{\prime}\right)\left(\Psi\left(\lambda z_{1} \ldots \lambda z_{n}\left((\mid(G, x), y), z_{1}\right), \ldots z_{n}\right)\right), \Psi\left(u_{1}\right), \ldots, \Psi\left(u_{k}\right)\right) \\
& =\Psi(F)\left(\lambda Z_{1} \ldots \lambda Z_{n} \Psi(G)\left(\Psi(x), \Psi(y), Z_{1}, \ldots, Z_{n}\right), \Psi\left(u_{1}\right), \ldots, \Psi\left(u_{k}\right)\right) \\
& =-H^{\prime}\left(\Psi(x), \Psi(y), \Psi\left(u_{1}\right), \ldots, \Psi\left(u_{k}\right)\right) ;
\end{aligned}
$$

thus $\boldsymbol{H}^{\prime}=\Psi(\boldsymbol{H})$ by $(37)$.

d. If $F \in \mathcal{R} \cap(\alpha(\beta(\beta \gamma))), \gamma=\left(\gamma_{1}\left(\ldots\left(\gamma_{n} 0\right)\right)\right), \Psi(F) \in \operatorname{Rec}, \quad D$ satisfies (28), and $H$ satisfies (34) with the substitution $F / \Psi(F)$, then $H=\Psi(D)$. (28), (37) and (34) imply that

$$
\begin{aligned}
\left(\left(((D, x), y), u_{1}\right), \ldots u_{n}\right) & =\left(\left((((F, x), y), y), u_{1}\right), \ldots u_{n}\right) \\
& =\Psi(F)\left(\Psi(x), \Psi(y), \Psi(y), \Psi\left(u_{1}\right), \ldots, \Psi\left(u_{n}\right)\right) \\
& =H\left(\Psi(x), \Psi(y), \Psi\left(u_{1}\right), \ldots, \Psi\left(u_{n}\right)\right),
\end{aligned}
$$

whence indeed $H=\Psi(D)$.

e. If $F \in \mathcal{R} \cap(\alpha \beta), G \in \mathcal{R} \cap(\alpha(0(\beta \beta))), \quad \beta=\left(\beta_{\mathbf{1}}\left(\ldots\left(\beta_{n} 0\right)\right)\right), H$ satisfies (29), $\Psi\left(F^{\prime}\right) \in \operatorname{Rec}, \Psi(G) \epsilon \operatorname{Rec}$, and $H^{\prime}$ satisfies (35) with the substitution $F / \Psi(F)$ and $G / \Psi(G)$, then $H^{\prime}=\Psi(H)$. Indeed, from (29), (37), (38) and (35) we obtain the equalities

$$
\begin{aligned}
& \left(\left(((H, t), 0), u_{1}\right), \ldots, u_{n}\right)=\left(\left((F, t), u_{1}\right), \ldots, u_{n}\right) \\
& =\Psi\left(F^{\prime}\right)\left(\Psi(t), \Psi\left(u_{1}\right), \ldots, \Psi\left(u_{n}\right)\right)=H^{\prime}\left(\Psi(t), 0, \Psi\left(u_{1}\right), \ldots, \Psi\left(u_{n}\right)\right), \\
& \left(\left(((H, t),(\mathcal{S}, n)), u_{1}\right), \ldots, u_{n}\right)=\left(\left(\left(((G, t, n),((H, t), n)), u_{1}\right), \ldots, u_{n}\right)=\right.
\end{aligned}
$$


$\left.\left.=\left(\left(((G, t), n), \lambda u_{1} \ldots \lambda u_{n}\left(((H, t), n), u_{1}\right), \ldots, u_{n}\right)\right), u_{1}\right), \ldots, u_{n}\right)$

$\left.=\Psi(G)\left(\Psi(t), \Psi(n), \Psi\left(\lambda u_{1} \ldots \lambda u_{n}\left(((H, t), n), u_{1}\right), \ldots, u_{n}\right)\right), \Psi\left(u_{1}\right), \ldots, \Psi\left(u_{n}\right)\right)$

$=\Psi(G)\left(\Psi(t), \Psi(n), \lambda U_{1} \ldots \lambda U_{n} \Psi(H)\left(\Psi(t), \Psi(n), U_{1}, \ldots, U_{n}\right), \Psi\left(u_{1}\right), \ldots, \Psi\left(u_{n}\right)\right)$

$=\Psi(G)\left(\Psi(t), n, \lambda U_{1} \ldots \lambda U_{n}\left(((H, t), n), \Psi^{-1}\left(U_{1}\right)\right), \ldots, \Psi^{-1}\left(U_{n}\right)\right)$,

$$
\left.\Psi\left(u_{1}\right), \ldots, \Psi\left(u_{n}\right)\right)
$$

hence, supposing that

$$
\left.\left(\left((H, t), n_{1}\right), u_{1}\right), \ldots, u_{n}\right)=H^{\prime}\left(\Psi(t), n, \Psi^{\prime}\left(u_{1}\right), \ldots, \Psi\left(u_{n}\right)\right),
$$

we obtain

$$
\begin{aligned}
& \left.\left(((H, t), n+1), u_{1}\right), \ldots, u_{n}\right) \\
& =\Psi(G)\left(\Psi(t), n, \lambda U_{1} \ldots \lambda U_{n} H^{\prime}\left(\Psi(t), n, U_{1}, \ldots, U_{n}\right), \Psi\left(u_{1}\right), \ldots, \Psi\left(u_{n}\right)\right) \\
& =H^{\prime}\left(\Psi(t), n+1, \Psi\left(u_{1}\right), \ldots, \Psi\left(u_{n}\right)\right) ;
\end{aligned}
$$

thus by induction we find that equality (39) is true for every $n \in N$; this means that $H^{\prime}=\Psi(H)$.

f. If $F \in R \cap(\alpha \beta), G \in \mathcal{R} \cap \alpha, \beta=\left(\beta_{1}\left(\ldots\left(\beta_{n} 0\right)\right)\right), \Psi(F) \in \operatorname{Rec}, \Psi(G)$ $\epsilon$ Rec, and $H$ satisfies (36) with the substitution $F / \Psi(F)$ and $G / \Psi(G)$, then $H=\Psi((F, G))$. Indeed (37) and (36) imply that

$$
\begin{aligned}
\left(\left((F, G), u_{1}\right), \ldots, u_{n}\right) & =\Psi(F)\left(\Psi(G), \Psi\left(u_{1}\right), \ldots, \Psi\left(u_{n}\right)\right) \\
& =\left(H \Psi\left(u_{1}\right), \ldots, \Psi\left(u_{n}\right)\right) ;
\end{aligned}
$$

hence $H=\Psi(F, G)$, which completes the inductive proof.

(The special cases for $n=0$ or $k=0$ are evident.) $\left({ }^{\circ}\right)$

\section{Comparision with Kleene's recursive functionals and} application to Gödel's interpretation of intuitionistical aritmetic. Theorem 5.2 shows that practically the recursive objects of Section 1 may be identified with the recursive functionals defined in Section 5 . The presentation of Section 5 is more convenient to compare our notion
with other notions of recursiveness.

(") Using the definition of primitive recursive functionals mentioned in footnote ( ${ }^{(8)}$ we obtain the analogon of the theorem 5.2 for the primitive recursive objects defined
in footnote $\left({ }^{8}\right)$. Cf. footnote $\left({ }^{7}\right)$.
Let $\mathrm{KR}$ be the class of primitive recursive functionals defined by Kleene in [5]. It is evident that

6.1. KR $\subset$ Rec.

Proof. The only difficulty is connected with the possibility of permutation of arguments. Let $\mathfrak{A}_{1}$ be a permutation of the $n$-tuple $\mathfrak{A}$ of arguments. If $\psi\left(\mathfrak{A}_{1}\right)$ belongs to Rec, then, if we take $\Psi^{-1}(\psi)$ according to 1.5, there exists an $f \in \Re$ such that $\left(\left(\Psi^{-1}(\psi), a_{1}\right), \ldots, a_{n}\right)=\left(\left(f, b_{1}\right), \ldots, b_{n}\right)$ where $b_{1}, \ldots, b_{n}$ is a permutation of $a_{1}, \ldots, a_{n}$ corresponding to the permutation of $\mathfrak{2}$ into $\mathfrak{A}_{1}$. Hence by 5.2 :

$$
\Psi(f)(\mathfrak{R})=\psi\left(\mathfrak{R}_{1}\right) .
$$

This remark immediately shows that the class Rec satisfies S1-S8 of Kleene. $\left({ }^{10}\right)$

But there is no restricted converse inclusion. Already in the domain of -functions on natural numbers the class Rec is larger than $K R$, because it contains functions defined by means of the multiple recursion schema while the class $\mathrm{KR}$ contains only primitive recursive functions on natural numbers. Let $G$ be the function defined by double recursion by R. M. Robinson in [8]:

$$
\begin{aligned}
& \text { (i) } \quad G(0, x)=A(x) \text {, } \\
& \text { (ii) } \quad G(\mathrm{Sn}, 0)=0 \text {, } \\
& \text { (iii) } G(\mathrm{Sn}, \mathrm{Sx})=G(n, B(x, G(\mathrm{Sn}, x))) \text {, }
\end{aligned}
$$

where $A$ and $B$ are some primitive recursive functions.

6.2. $G \in \operatorname{Rec}$ and $G \notin \mathrm{KR}$.

Proof. The second part of 6.2 follows from Kleene's Theorem VIII of [5], because $G$ is not primitive recursive, as was proved by R. M. Robinson in [8].

We construct the function $G$ in the following way. We start by $I(\alpha, x)=\alpha(x)$. Using $(31),(36)$, and $\left(32^{\prime}\right)$ we obtain $I^{4}$ such that

(41) $\quad I^{4}(x, \alpha)=I^{3}(0, x, \alpha)=I^{2}(v, x, \alpha)=I^{1}(v, \alpha, x)=I(\alpha, x)=\alpha(x)$, then by means of $\left(33^{\prime \prime}\right)$ we obtain $f$ such that

$$
f(x, y, \alpha)=I^{4}(B(x, y), \alpha) ;
$$

we permute the arguments in the same way as in (41), and we obtain $y$ such that

$$
g(\alpha, x, y)=f(x, y, \alpha)
$$

$\left({ }^{10}\right) \mathrm{KR}$ are also primitive recursive in the sense determined in footnote ( $\left.{ }^{8}\right)$ but they contain a smaller wealth of types. 
now by the induction schema $\left(35^{\prime}\right)$ we define $H$ such that

$$
\begin{aligned}
H(\alpha, 0) & =0^{3}(\alpha)=0^{2}(0, \alpha)=0^{1}(x, \alpha)=0\left(I^{4}(x, \alpha)\right)=0, \\
H(\alpha, \mathrm{Sx}) & =g(\alpha, x, H(\alpha, x)) .
\end{aligned}
$$

Formulas (41)-(44) imply that

$$
H(\alpha, 0)=0, \quad H(\alpha, \mathrm{Sx})=\alpha(B(x, H(\alpha, x))) .
$$

Next, using (31), we obtain $H$ such that

$$
H^{2}(l, n, x, a)=H^{1}(n, \alpha, x)=H(\alpha, x) .
$$

The most essential is the following definition by means of (35):

$$
\begin{aligned}
G^{\prime}(t, 0, x) & =A^{\prime}(t, x)=A(x), \\
G^{\prime}(t, \mathrm{Sn}, x) & =H^{2}\left(t, n, \lambda_{x} G^{\prime}(t, n, x), x\right)=H\left(\lambda_{i} x\left(t^{\prime}(t, n, x), x\right) .\right.
\end{aligned}
$$

Then we can prove that $G(n, x)=G^{\prime}(0, n, x)$ satisties (40). (i) is ovident, (ii) follows immediately from (45)-(47). To prove (iii) from (47) and (45) we obtain

$$
\begin{aligned}
G(\mathrm{Sn}, \mathrm{Sx}) & =H\left(\lambda_{x} G^{\prime}(0, n, x), \mathrm{Sx}\right) \\
& =\lambda_{x} G^{\prime}(0, n, x)\left(B\left(x, H\left(\lambda x G^{\prime}(0, n, x), x\right)\right)\right) \\
& =G\left(0, n,\left(B\left(x, H\left(\lambda x G^{\prime}(0, n, x), x\right)\right)\right)\right) \\
& =G\left(n,\left(B\left(x, H\left(\lambda x G^{\prime}(0, n, x), x\right)\right)\right)\right) .
\end{aligned}
$$

(iii) follows from (47) and (48).

On the other hand, the class $\mathcal{R}$ of recursive objects may be considered as identical with the class of recursive functionals mentioned by Gödel [3] and Kreisel [6]. (They also remarked that the functional recursive schema (in our notation (11)) includes "all ordinal recursive functionals of order $<\varepsilon_{0}$, while Kleene's functionals are only primitive recursive".) (11)

The class $\mathscr{R}$ of recursive objectis is convenient for an exact formulation of Gödel's interpretation of intuitionistic axithmetics. Primitive recursive functional are unsufficient for this purpose $\left(^{12}\right)$. We need to have the functional recursion schema (11), which implies the multiple recursion $(35)\left({ }^{13}\right)$. Using our recursive objects, wo may describe Gödel's interpretation as follows.

(11) See Kreisel [6], p. 110 and 112. However, it seems to me that Kreisel's argument presents rather an open problem.

(12) For example, the formula $\bigwedge x z \bigvee y \Phi(x, z, y)$ (where $\Phi$ is an arithmetic formula strongly representing the function $G$ defined in $(40))$ is not primitively reoursively true in the sense clearly analogous to 6.3 .
Let HAr be the system of Heyting's elementary arithmetic of natural numbers. First we define a translation $T$ of the formulas of $\mathrm{HAr}$ into the formulas of AT: the arithmetic with the whole simple theory of types formulated by means of the notion $(\ldots, \ldots)$ of application. The translation $T$ refer to all formulas of AT. (The formulas of HAr may be considered as belonging also to the formulas of AT.) Every variable $V_{\beta}^{\alpha}$ of AT has a type-index $\beta$ and a shape-index $\alpha$. Both indexes are numerals. We begin by the definition of the translation $T^{\prime}$ for the formulas of the form:

(50) $F=$ [The matrix $M$ of quantifiers] (the formula $A$ without quantifiers).

We assume that $T^{\prime \prime}(F)=F$ if $M$ contains at most one quantifier. Now suppose that the function $T^{\prime}$ is defined for all formulas of the form (50) with the number of quantifiers $\leqslant n$; if the formula $F$ having $n+1$ quantifiers begins with an existential quantifier $F=\vee v_{\beta}^{\alpha} A$, then we put

$$
T^{\prime}\left(F^{\prime}\right)=\bigvee v_{\beta}^{\alpha} T^{\prime}(A) ;
$$

if the formula $F$ begins with a general quantifier $F=\wedge v_{\beta}^{a} A$, we put

$T^{\prime}\left(F^{\prime}\right)=\bigwedge v_{\beta}^{\alpha} T^{\prime}(A) \quad$ if $T^{\prime}(A)$ begins also with a general quantifier,

$T^{\prime}\left(F^{\prime}\right)=\bigvee v_{(\beta \delta)}^{\gamma}, T^{\prime}\left(\Lambda v_{\beta}^{\alpha} B\left(v_{\beta}^{\alpha},\left(v_{(\beta \delta)}^{\gamma}, v_{\beta}^{\alpha}\right)\right)\right) \quad$ if $\quad T^{\prime}(A)=\vee v_{\delta}^{\gamma} B\left(v_{\beta}^{\alpha}, v_{\delta}^{\gamma}\right)$.

Now we define the translation $T$ :

$$
T(F)=F \quad \text { if } F \text { is anatomic formula. }
$$

Now suppose that $E$ and $F$ are two formulas and the function $T$ is already defined for $E$ and $F$ and

$$
\begin{aligned}
& T(E)=\bigvee v_{\beta_{1}}^{a_{1}} \ldots \vee v_{\beta_{n}}^{\alpha_{n}} \wedge v_{\delta_{1}}^{\gamma_{1}} \ldots \wedge v_{\delta_{k}}^{\gamma_{k}} A, \\
& T(E)=\bigvee v_{\mathbf{e}_{1}}^{\pi_{1}} \ldots \vee v_{e_{m}}^{\tau_{m}} \wedge v_{\tau_{1}}^{\sigma_{1}} \cdots \wedge v_{\tau_{t}}^{\sigma_{t}} B,
\end{aligned}
$$

where $A$ and $B$ do not contain any quantifier; then (14)

$$
T(\neg E)=T^{\prime}\left(\wedge v_{\beta_{1}}^{\alpha_{1}} \ldots \wedge v_{\beta_{n}}^{a_{n}} \bigvee v_{\delta_{1}}^{\gamma_{1}} \ldots \vee v_{\delta_{k}}^{\gamma_{k}} \neg A\right),
$$

$T(E \wedge F)=T^{\prime}\left(\bigvee v_{\beta_{1}}^{a_{1}} \cdots \vee v_{\beta_{n}}^{a_{n}} \bigvee v_{\Omega_{1}}^{\pi_{1}} \ldots \vee v_{\Omega_{m}}^{n_{m}} \wedge v_{\delta_{1}}^{\gamma_{1}} \cdots \wedge v_{\delta_{k}}^{\gamma_{k}} \wedge v_{\tau_{1}}^{\sigma_{1}} \ldots \wedge v_{\tau_{t}}^{\sigma_{t}}(A \wedge B)\right)$,

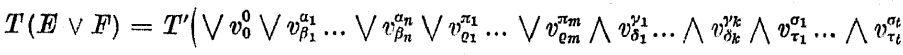

$$
\left.\left(\left(A \wedge v_{0}^{0}=0\right) \wedge\left(B \wedge v_{0}^{0}=1\right)\right)\right),
$$

$T\left(E \rightarrow E^{\prime}\right)=T^{\prime}\left(\wedge v_{\beta_{1}}^{a_{1}} \ldots \wedge v_{\beta_{n}}^{\alpha_{n}} \vee v_{e_{1}}^{\pi_{1}} \ldots \vee v_{e_{m}}^{\pi_{m}} \wedge v_{r_{1}}^{\sigma_{1}} \ldots \wedge v_{\tau_{t}}^{\pi_{t}} \vee v_{\delta_{1}}^{\gamma_{1}} \ldots \vee v_{\delta_{k}}^{\gamma_{k}}(A \rightarrow B)\right)$,

$T\left(\bigvee v_{\theta}^{\varepsilon} E\right)=T^{\prime}\left(\bigvee v_{\theta}^{\varepsilon} T(E)\right)$,

$T\left(\bigwedge v_{\theta}^{\varepsilon} E\right)=T^{\prime}\left(\bigwedge v_{\theta}^{\varepsilon} T(E)\right)$.

${ }^{(18)}$ In the sense of R. Péter [7].

(14) If some quantifiers fall out of the representation of $T^{\prime}(E)$ or $T(E)$, they also fall out of the representation of $T(7 E), T(E \wedge F)$ and so on 
Hence for every formula $F^{\text {H }}$ of $A T$ the translation has the form

$$
T(F)=\bigvee v_{\beta_{1}}^{\alpha_{1}} \ldots \bigvee v_{\beta_{n}}^{a_{n}} \wedge v_{\delta_{1}}^{\gamma_{1}} \ldots \wedge v_{\delta_{k}}^{\gamma_{k}} A
$$

where $A$ does not contain any quantifier.

Gödel's interpretation of intuitionistic arithmetic may be formulated in the following semantical way:

6.3 (The definition of recursive truth). If If satisfying (51) is a closed formula of $\mathrm{AT}$, then we shall say that $F$ is recursively true if and only if there exist $\nabla_{\beta_{1}}^{\alpha_{1}}, \ldots, V_{\beta_{n}}^{\alpha_{n}} \in \mathcal{R}$ of types indexed by $\beta_{1}, \ldots, \beta_{n}$ such that they satisfy the formula

$$
\bigwedge v_{\delta_{1}}^{\gamma_{1}} \ldots \wedge v_{\delta_{k}}^{\gamma_{k}} A
$$

in the standard model of ArI.

6.4 (The theorem on the recursive tiruth of HAr). If If is a theorem of HAr, then $T\left(F^{\prime}\right)$ is recursively true.

The proof consists of verifying all axioms and rules of inference of HAr. The calculations are all fairly easy. The recuuired recursive objects may be directly constructed from the injtial elements of the class $\chi$. To verify the induction rule (proving 6.4) we need the simultaneous induction schema, but according to 3.2 the class $\%$ is closed under this kind of recursion. To verify the propositional rules

$$
\begin{gathered}
(p \rightarrow g) \rightarrow((g \rightarrow r) \rightarrow(p \rightarrow r)), \\
((p \rightarrow q) \wedge(r \rightarrow q)) \rightarrow((p \vee r) \rightarrow q)
\end{gathered}
$$

we need to make use of the following property 6.5 of the class $\%$ :

6.5 (Conditional definability). If $f, g, j \in \Re, f, g \in\left(\alpha_{1}\left(\ldots\left(\alpha_{n} \beta\right)\right)\right)$ and $j \in\left(\alpha_{1}\left(\ldots\left(\alpha_{n} 0\right)\right)\right)$, then there exists an $h \in \mathcal{R} \cap\left(\alpha_{1}\left(\ldots\left(\alpha_{n} \beta\right)\right)\right)$ such that

(52) $\left(\left(h, x_{1}\right), \ldots, x_{n}\right)=\left\{\begin{array}{lll}\left(\left(f, x_{1}\right), \ldots, x_{n}\right) & \text { when } & \left(\left(j, x_{1}\right), \ldots, x_{n}\right)=0, \\ \left(\left(g, x_{1}\right), \ldots, x_{n}\right) & \text { when } & \left(\left(j, x_{1}\right), \ldots, x_{n}\right) \neq 0 .\end{array}\right.$

This property results from 1.5 and 2.5 in the following way. Let $\beta=\left(\beta_{1}\left(\ldots\left(\beta_{k} 0\right)\right)\right)$; then $(52)$ implies that

$$
\begin{gathered}
\left(\left(\left(\left(h, x_{1}\right), \ldots, x_{n}\right), y_{1}\right), \ldots, y_{k}\right)=\left(\left(\left(\left(f, x_{1}\right), \ldots, x_{n}\right), y_{1}\right), \ldots, y_{k}\right) \cdot\left(1-\left(\left(j, x_{1}\right), \ldots, x_{n}\right)\right)++ \\
+\left(\left(\left(\left(g, x_{1}\right), \ldots, x_{n}\right), y_{1}\right), \ldots, y_{k}\right) \cdot\left(1-\left(1-\left(\left(j, x_{1}\right), \ldots, x_{n}\right)\right)\right) .
\end{gathered}
$$

Hence 2.5 and 1.5 imply that $h \in R$. (15)

${ }^{(18)}$ The aim of this paper has been only to point out the definition of tlie class $" 2$ ? (stated at the beginning) which seems to be elegant, and to prove 3.2 (simultaneous recursion theorem) in order to make easier a precise proof of Gödels' interpretation theorem.

\section{References}

[1] H. B. Curry and R. Feys, Combinatory logic, Studies in logic and the foundations of mathematics, Amsterdam 1958.

[2] A. Church, A formulation of the simple theory of types, Journal of Symbolic Logic 5 (1940), pp. 56-68.

[3] K. Gödel, Über eine bisher noch nicht benützte Erweiterung des finiten Standpunkten, Dialectica 47-48, vol. 12 (1958), pp. 280-287.

[4] S. C. Kleene, On the interpretation of intuitionistio number theory, Journal of Symbolic Logic 10 (1945), pp. 109-124.

[5] - Recursive functionals and quantifiers of finite types $I$, Transactions of the American Math. Soc. 91 (1959), pp. 1-52.

[6] $\mathrm{G}$. Kreisel, Interpretation of analysis by means of constructive functionals of finite types. Constructivity in Mathematics, A. Heyting (editor). Studies in Logic and the Foundations of Mathematics, Amsterdam 1959, pp. 101-128.

[7] R. Péter, Rekursive Funktionen, Budapest 1951.

[8] R. M. Robinson, Recursion and double recursion, Bull. Amer. Math. Soc. 54 (1948), pp. 987-993.

[9] - Primitive recursive functions, Bull. Amer. Math. Soc. 53 (1947), pp. 925-942.

Reçu par la Rédaction le 27.11.1962 